Acta Crystallographica Section A

Foundations of Crystallography

ISSN 0108-7673

Received 26 May 2006

Accepted 6 July 2006

(C) 2006 International Union of Crystallography Printed in Great Britain - all rights reserved

\section{On the calculation of the electrostatic potential, electric field and electric field gradient from the aspherical pseudoatom model}

\author{
Anatoliy Volkov, ${ }^{\text {a* Harry F. King, }}{ }^{\text {a Philip Coppens }}{ }^{\mathrm{a}}$ and Louis J. Farrugia ${ }^{\mathrm{b}}$ \\ ${ }^{a}$ Department of Chemistry, University at Buffalo, State University of New York, Buffalo, \\ NY 14260-3000, USA, and 'bestCHEM, Department of Chemistry, University of Glasgow, \\ Glasgow G12 8QQ, UK. Correspondence e-mail: volkov@chem.buffalo.edu
}

\begin{abstract}
Accurate, yet simple and efficient, formulae are presented for calculation of the electrostatic potential (ESP), electric field (EF) and electric field gradient (EFG) from the aspherical Hansen-Coppens pseudoatom model of electron density [Hansen \& Coppens (1978). Acta Cryst. A34, 909-921]. They are based on the expansion of $\left|\mathbf{r}^{\prime}-\mathbf{r}\right|^{-1}$ in spherical harmonics and the incomplete gamma function for a Slater-type function of the form $R_{l}(r)=r^{n} \exp (-\alpha r)$. The formulae are valid for $0 \leq r \leq \infty$ and are easily extended to higher values of $l$. Special treatment of integrals is needed only for functions with $n=l$ and $n=l+1$ at $r=0$. The method is tested using theoretical pseudoatom parameters of the formamide molecule obtained via reciprocal-space fitting of $\mathrm{PBE} / 6-31 \mathrm{G}^{* *}$ densities and experimental X-ray data of $\mathrm{Fe}(\mathrm{CO})_{5}$. The ESP, EF and EFG values at the nuclear positions in formamide are in very good agreement with those directly evaluated from density-functional PBE calculations with 6-31G**, aug-cc-pVDZ and aug-cc-pVTZ basis sets. The small observed discrepancies are attributed to the different behavior of Gaussian- and Slater-type functions near the nuclei and to imperfections of the reciprocal-space fit. An EF map is displayed which allows useful visualization of the lattice EF effects in the crystal structure of formamide. Analysis of experimental $100 \mathrm{~K} \mathrm{X}$-ray data of $\mathrm{Fe}(\mathrm{CO})_{5}$ yields the value of the nuclear quadrupole moment $Q\left({ }^{57} \mathrm{Fe}^{m}\right)=0.12 \times 10^{-28} \mathrm{~m}^{2}$ after taking into account Sternheimer shielding/antishielding effects of the core. This value is in excellent agreement with that reported by Su \& Coppens [Acta Cryst. (1996), A52, 748-756] but slightly smaller than the generally accepted value of $0.16 \pm 5 \% \times 10^{-28} \mathrm{~m}^{2}$ obtained from combined theoretical/spectroscopic studies [Dufek, Blaha \& Schwarz (1995). Phys. Rev. Lett. 25, 3545-3548].
\end{abstract}




$$
\begin{aligned}
V(\mathbf{r}) & =V^{\mathrm{nuc}}(\mathbf{r})+V^{\mathrm{elec}}(\mathbf{r})=\left(\sum_{i} V_{i}^{\mathrm{nuc}}(\mathbf{r})\right)+V^{\mathrm{elec}}(\mathbf{r}) \\
& =\sum_{i=1}^{N} \frac{Z_{i}}{\left|\mathbf{R}_{i}-\mathbf{r}\right|}-\int \frac{\rho\left(\mathbf{r}^{\prime}\right)}{\left|\mathbf{r}^{\prime}-\mathbf{r}\right|} \mathrm{d}^{3} \mathbf{r}^{\prime}
\end{aligned}
$$

where $i=1, \ldots, N$ is the index of the nucleus located at $\mathbf{R}_{i}$ carrying positive charge $Z_{i}$. The second term represents the contribution of the continuous distribution of the negatively charged electron density $\rho(\mathbf{r})$. When calculating $V(\mathbf{r})$ at any point in space where $\mathbf{r} \neq \mathbf{R}_{i}$, both terms need to be taken into account, while, at $\mathbf{r}=\mathbf{R}_{i}$, the contribution of the nuclear potential of the $i$ th nucleus (located exactly at $\mathbf{R}_{i}$ ) must be omitted. The calculation of the nuclear potential and its derivatives is trivial and need not be described here. It is the calculation of the electronic potential $V^{\text {elec }}(\mathbf{r})$ that presents more problems.

Various methods for calculating the ESP from X-ray diffraction data have been described and consequently applied in the literature. These methods can basically be split into two very different groups: (i) directly from experimentally measured structure factors (Bertaut, 1978; Stewart, 1979; Schwarzenbach \& Thong, 1979) and (ii) from static models of electron density. Discussion of methods belonging to group (i) lies outside the scope of this paper. More important is the second group, which allows the calculation of electrostatic properties of atoms and molecules from the electron densities deconvoluted from nuclear motions. These are relatively unaffected by Fourier-series termination and allow a direct comparison with theoretical results. These methods are based on so-called pseudoatom charge-density models.

By far the most widely used aspherical pseudoatom formalism is based on the Hansen-Coppens multipolar model of ED (Coppens, 1997; Hansen \& Coppens, 1978). In this formalism, the electron density at each point in space $\rho(\mathbf{r})$ is described by a superposition of atomic like densities $\rho_{\text {at }}(\mathbf{r})$, called pseudoatoms:

$$
\rho(r)=\sum \rho_{\mathrm{at}}(r)
$$

Each pseudoatom is modeled using the modified Laplace series:

$$
\begin{aligned}
\rho_{\mathrm{at}}(r)= & P_{\text {core }} \rho_{\text {core }}(r)+P_{\mathrm{val}} \kappa^{3} \rho_{\mathrm{val}}(\kappa r) \\
& +\sum_{l=0}^{l_{\max }} \kappa^{\prime 3} R_{l}\left(\kappa^{\prime} r\right) \sum_{m=0}^{l} P_{l m \pm} d_{l m \pm}(\Omega) .
\end{aligned}
$$

The first and second terms of expansion are the spherically averaged Hartree-Fock core and valence densities (Clementi $\&$ Roetti, 1974). The population of the core, $P_{\text {core, }}$ is always frozen, while the population of the spherical valence shell $P_{\text {val }}$ is refined together with the expansion-contraction parameter $\kappa$. The third term describes the aspherical deformation density. Coefficients $P_{l m \pm}$ are the population parameters and $\kappa^{\prime}$ are the dimensionless adjustment coefficients of the radial functions $R_{l}$. In equation (8), $r$ is the distance from the pseudoatom center, $r=|\mathbf{r}-\mathbf{R}|$, and $\Omega$ are the corresponding angular coordinates. The angular factor $d_{l m \pm}$ is a real densitynormalized spherical harmonic:

$$
d_{l m \pm}(\Omega)=D_{l m} y_{l m \pm}(\Omega)
$$

where $m \geq 0$ and $y_{l m \pm}$ is the normalized linear combination of complex spherical harmonics:

$$
\begin{aligned}
& y_{00}=Y_{00} \\
& y_{l m+}=(-1)^{m}\left(Y_{l m}+Y_{l,-m}\right) / \sqrt{2}, \quad m>0 \\
& y_{l m-}=(-1)^{m}\left(Y_{l m}-Y_{l,-m}\right) / \sqrt{-2}, \quad m>0 \text {. }
\end{aligned}
$$

Renormalization factors $D_{l m}$ are given by Paturle \& Coppens (1988) and Coppens (1997).

Calculation of the electrostatic potential and its first and second derivatives, i.e. negatives of electric field and electric field gradient, respectively, from the pseudoatom model is not straightforward. It was shown that the electrostatic potential can be evaluated from the pseudoatom model in various ways, e.g. directly from $\rho(\mathbf{r})$ or from its truncated Fourier-series expansion (Brown \& Spackman, 1994).

The method of Su \& Coppens $(1992,1996)$ is based on the Fourier convolution theorem previously applied by Epstein \& Swanton (1982) to a calculation of the EFG. While it does not contain approximations and is formally exact at any point in space, it involves the evaluation of fairly complicated oneelectron two-center integrals. This method was encoded in the computer program MOLPROP and later included in the experimental charge-density package $X D$ (Koritsanszky et al., 2003). However, the program does not always reproduce the correct results when theoretical structure factors are used to evaluate the physical properties. It is not clear whether this is due to programming errors or errors in the derivation of twocenter integrals [labeled as $A_{N, l_{1}, l_{2}, k}(Z, R)$ in the original paper]. Note that the method of $\mathrm{Su} \&$ Coppens only allows calculation of the traceless EFG tensor at the nuclear positions.

Several methods for the calculation of the ESP/EFG were proposed by Brown \& Spackman (1994). The direct-space evaluation of the EFG, also based on the Fourier transform theorem, is closely related to that of $\mathrm{Su} \&$ Coppens. It was included in the experimental charge-density package VALRAY (Stewart et al., 1998). These authors also presented the combined Fourier/direct-space evaluation of the EFG, as well as calculation of the EFG via numerical differentiation of the ESP. While giving more or less reasonable results, these methods are either too computationally demanding or have convergence problems with Fourier sums.

Ghermani, Bouhmaida, Lecomte and co-workers (Lecomte et al., 1992; Ghermani, Bouhmaida \& Lecomte, 1993; Ghermani, Lecomte \& Bouhmaida, 1993; Ghermani et al., 1994; Bouhmaida et al., 1997) reported expressions for the ESP derived directly from the Hansen-Coppens density model [equation (8)], in which the ESP due to a pseudoatom is expanded in the same way as the density $\rho_{\text {at }}(\mathbf{r})$ itself [equation (8)], i.e.

$$
V_{\mathrm{at}}(\mathbf{r})=V_{\text {core }}(r)+V_{\text {val }}(r)+\Delta V(\mathbf{r}) .
$$


Here $V_{\text {core }}(r), V_{\text {val }}(r)$ and $\Delta V(\mathbf{r})$ are the spherical core, spherical valence and aspherical deformation contributions, respectively, and are given by

$$
\begin{aligned}
V_{\text {core }}(\mathbf{r})= & \frac{Z}{|\mathbf{r}-\mathbf{R}|}-\int_{0}^{\infty} \frac{\rho_{\text {core }}\left(r^{\prime}\right)}{\left|\mathbf{r}-\mathbf{R}-\mathbf{r}^{\prime}\right|} \mathrm{d}^{3} \mathbf{r}^{\prime} \\
V_{\mathrm{val}}(r)= & -\int_{0}^{\infty} \frac{P_{\mathrm{val}} \kappa^{\prime 3} \rho_{\mathrm{val}}\left(\kappa^{\prime} r^{\prime}\right)}{\left|\mathbf{r}-\mathbf{R}-\mathbf{r}^{\prime}\right|} \mathrm{d}^{3} \mathbf{r}^{\prime}, \\
\Delta V(\mathbf{r})= & -4 \pi \sum_{l m} \frac{\kappa^{\prime} P_{l m}}{2 l+1}\left[\frac{1}{\kappa^{\prime l+1}|\mathbf{r}-\mathbf{R}|^{l+1}} \int_{0}^{\kappa^{\prime}|\mathbf{r}-\mathbf{R}|} t^{l+2} R_{l}(t) \mathrm{d} t\right. \\
& \left.+\kappa^{\prime l}|\mathbf{r}-\mathbf{R}|^{l} \int_{\kappa^{\prime}|\mathbf{r}-\mathbf{R}|}^{\infty} \frac{R_{l}(t)}{t^{l-1}} \mathrm{~d} t\right] d_{l m}(\Omega)
\end{aligned}
$$

where $\mathbf{R}$ is the position of the nucleus and $\mathbf{r}^{\prime}$ is the position vector relative to $\mathbf{R}$ [unlike that in equation (3)]. Note that, in expression (12), the contribution of the nuclear potential is explicitly combined with the core-electron contribution. Formula (14) was derived using Green's function and the property of orthogonality of the spherical harmonic functions and is valid for any point in space, since no approximations are used. These formulae were implemented in the program ELECTROS (Ghermani et al., 1992) derived from the earlier program MOLPOT (He, 1983). Numerous studies have been published using this approach.

In the current paper, we review the derivation of equation (14), providing more detail than available in the literature. In particular, we call attention to practical problems associated with numerical instabilities, and present simple stable expressions for the ESP, EF and EFG that overcome these problems. We apply our methods in a theoretical study of the $\mathrm{EF}$ in the crystal structure of formamide and in a determination of the ${ }^{57} \mathrm{Fe}^{m}$ nuclear quadrupolar moment from experimental X-ray diffraction data.

\section{Calculation of the electronic potential and its derivatives for Slater-type functions}

Each term in the pseudoatom formalism (8) can be reduced to a linear combination of Slater-type (Slater, 1932) density basis functions with the general form

$$
\zeta(r)=R(r) d_{l m \pm}(\Omega)=N(n, \alpha) r^{n} \exp (-\alpha r) d_{l m \pm}(\Omega),
$$

where $\alpha$ is the effective exponent and $N(n, \alpha)$ is the normalization factor (Coppens, 1997). The corresponding electrostatic potential is

$$
V_{\zeta}(\mathbf{r})=-\int \frac{\zeta\left(\mathbf{r}^{\prime}\right)}{\left|\mathbf{r}^{\prime}-\mathbf{r}\right|} \mathrm{d}^{3} \mathbf{r}^{\prime}
$$

$\left|\mathbf{r}^{\prime}-\mathbf{r}\right|^{-1}$ can be expanded in real spherical harmonics (Jackson, 1975):

$$
\frac{1}{\left|\mathbf{r}^{\prime}-\mathbf{r}\right|}=\sum_{l} \frac{4 \pi}{2 l+1} \frac{r_{<}^{l}}{r_{>}^{l+1}} \sum_{m} y_{l m \pm}(\Omega) y_{l m \pm}\left(\Omega^{\prime}\right),
$$

where $r_{<}$is the smaller and $r_{>}$is the greater of $|\mathbf{r}-\mathbf{R}|$ and $\left|\mathbf{r}^{\prime}-\mathbf{R}\right|$. Note that the $y_{l m \pm}$ are obtained by unitary transformation of the $Y_{l m}$, which implies that the form of the spherical harmonic addition theorem (Edmonds, 1974) is preserved, i.e.

$$
\sum_{m=-l}^{l} Y_{l m}^{*}(\Omega) Y_{l m}\left(\Omega^{\prime}\right)=\sum_{m=0}^{l} y_{l m \pm}(\Omega) y_{l m \pm}\left(\Omega^{\prime}\right) .
$$

When (15) and (17) are inserted into (16), the only term in the double sum that survives after integration over $\Omega^{\prime}$ is that term for which the $l m \pm$ indices match those in $\zeta(\mathbf{r})$. This follows immediately from orthonormality of the $y_{l m \pm}$ functions. Thus,

$$
V_{\zeta}(\mathbf{r})=\frac{4 \pi}{2 l+1} N(n, \alpha) d_{l m \pm}(\Omega) \int_{0}^{\infty} \frac{r_{<}^{l}}{r_{>}^{l+1}}\left(r^{\prime}\right)^{n+2} \exp \left(-\alpha r^{\prime}\right) \mathrm{d} r^{\prime}
$$

The radial integral in (15) splits into two terms:

$$
\begin{aligned}
I(n, l, \alpha r)= & \int_{0}^{\infty} \frac{r_{<}^{l}}{r_{>}^{l+1}} r^{n+2} \exp (-\alpha r) \mathrm{d} r \\
= & \frac{1}{|\mathbf{r}-\mathbf{R}|^{l+1}} \int_{0}^{|\mathbf{r}-\mathbf{R}|} r^{n+l+2} \exp (-\alpha r) \mathrm{d} r \\
& +|\mathbf{r}-\mathbf{R}|^{l} \int_{|\mathbf{r}-\mathbf{R}|}^{\infty} r^{n+1-l} \exp (-\alpha r) \mathrm{d} r \\
= & I_{1}(n, l, \alpha r)+I_{2}(n, l, \alpha r),
\end{aligned}
$$

so expression (19) becomes simply

$$
V_{\zeta}(\mathbf{r})=\frac{4 \pi}{2 l+1} N(n, \alpha) d_{l m \pm}(\Omega) I(n, l, \alpha r)
$$

Essentially the same formula is given by Ghermani, Bouhmaida, Lecomte and co-workers (Lecomte et al., 1992; Ghermani et al., 1992; Ghermani, Lecomte \& Bouhmaida, 1993; Ghermani, Bouhmaida \& Lecomte, 1993; Ghermani et al., 1994; Bouhmaida et al., 1997). Integrals $I_{1}(n, l, \alpha r)$ and $I_{2}(n, l, \alpha r)$ have the form of the incomplete gamma function (te Velde, 1990; te Velde et al., 2001):

$I_{1}(n, l, \alpha r)=\frac{(n+l+2) !}{\alpha^{n+2}} \frac{1}{(\alpha r)^{l+1}}\left[1-\exp (-\alpha r) \sum_{i=0}^{n+l+2} \frac{(\alpha r)^{i}}{i !}\right]$,

$I_{2}(n, l, \alpha r)=\frac{(n-l+1) !}{\alpha^{n+2}}(\alpha r)^{l} \exp (-\alpha r) \sum_{i=0}^{n-l+1} \frac{(\alpha r)^{i}}{i !}$.

The exponential terms in equations (22) and (23) represent the effects of the interpenetration of the charge-density distributions. The remaining term in equation (22) is the radial factor for the potential of a point multipole. These expressions are satisfactory for large values of $\alpha r$ but their straightforward evaluation for small values of $\alpha r$ results in severe numerical 
Table 1

Number of significant digits lost in direct evaluation of formulas (22) and (23).

\begin{tabular}{lllllr}
\hline & $l$ & & & & \\
\cline { 2 - 6 }$\alpha r$ & 0 & 1 & 2 & 3 & 4 \\
\hline 0.25 & 1 & 4 & 6 & 9 & 12 \\
0.50 & 1 & 2 & 5 & 8 & 9 \\
0.75 & 0 & 3 & 4 & 6 & 9 \\
1.00 & 0 & 2 & 4 & 5 & 7 \\
1.25 & 0 & 1 & 3 & 5 & 7 \\
1.50 & 1 & 2 & 1 & 4 & 6 \\
\hline
\end{tabular}

round-off errors as illustrated in Table 1. Numerical errors are relatively insensitive to $n$ but grow rapidly with increasing $l$ and decreasing $\alpha r$. The corresponding errors for first and second derivatives required for evaluation of EF and EFG are significantly greater than those for the ESP. The numerical errors are entirely due to the term $I_{1}(n, l, \alpha r)$, and can be avoided by rearranging equation (22):

$$
\begin{aligned}
I_{1}(n, l, \alpha r) & =\frac{(n+l+2) !}{\alpha^{n+2}} \frac{1}{(\alpha r)^{l+1}} \exp (-\alpha r) \sum_{i=n+l+3}^{\infty} \frac{(\alpha r)^{i}}{i !} \\
& =\frac{(n+l+2) !}{\alpha^{n+2}} \exp (-\alpha r) \sum_{i=n+2}^{\infty} \frac{(\alpha r)^{i}}{(i+l+1) !}
\end{aligned}
$$

This is a well behaved expression for any $r$. The rate of convergence of this infinite series is reported in Table 2. Thus, expression (24) is recommended for use for 'small' values of $\alpha r$ while expression (22) is preferred for medium and large values of $\alpha r$ as it converges more rapidly in that case.

Alternatively, integral $I(n, l, \alpha r)$ can be replaced with its Taylor-series expansion:

$$
\begin{aligned}
I(n, l, \alpha r)= & \frac{1}{\alpha^{n+2}}\left[(n-l+1) !(\alpha r)^{l}\right. \\
& \left.-(\alpha r)^{n+2} \sum_{k=0}^{\infty} \frac{(-\alpha r)^{k}(2 l+1)}{k !(k+n-l+2)(k+n+l+3)}\right] .
\end{aligned}
$$

Table 2 reports the number of terms required to achieve given accuracy when computing both $I(n, l, \alpha r)$ and its derivatives. Errors are relatively insensitive to $l$ and $n$.

Formula (21) can be directly applied to the deformation part of the pseudoatom expansion because the spherical harmonics of deformation functions are already density normalized (Coppens, 1997). The spherical core and valence densities are, however, calculated from products of wavefunction-normalized Slater functions. Nevertheless, the product of two Slater functions on the same center is still a Slater function:

$$
\begin{aligned}
N_{1} r^{n_{1}} & \exp \left(-\alpha_{1} r\right) N_{2} r^{n_{2}} \exp \left(-\alpha_{2} r\right) \\
& =\left(N_{1} N_{2}\right)\left(r^{n_{1}+n_{2}}\right) \exp \left[-\left(\alpha_{1}+\alpha_{1}\right) r\right] \\
& =N_{3} r^{n_{3}} \exp \left(-\alpha_{3} r\right),
\end{aligned}
$$

therefore, formula (21) can still be applied.
Table 2

Number of terms in equations (24) and (25) needed to achieve given accuracy.

\begin{tabular}{lll}
\hline & \multicolumn{2}{l}{ Number of significant digits } \\
\cline { 2 - 3 }$\alpha r$ & 10 & 15 \\
\hline 0.25 & 10 & 13 \\
0.50 & 12 & 16 \\
0.75 & 14 & 18 \\
1.00 & 15 & 20 \\
1.25 & 17 & 21 \\
1.50 & 18 & 23 \\
\hline
\end{tabular}

The first (EF) and second (EFG) derivatives of the ESP are then obtained by straightforward differentiation of expression (21).

\section{Calculation of the electronic potential and its derivatives near $r=0$}

Functions $d_{l m \pm}(\Omega)$ and their derivatives are poorly behaved near the origin, as they contain direction cosines, so we remove a factor $r^{l}$ from $I(n, l, \alpha r)$ and incorporate it into the angular factor:

$$
V_{\zeta}(r)=\frac{4 \pi}{2 l+1} N(n, \alpha)\left[d_{l m \pm}(\Omega) r^{l}\right]\left[r^{-l} I(n, l, \alpha r)\right] .
$$

Note that all terms in (25) contain powers of $\alpha r$ of $l$ or higher. For $l \leq 4$, derivatives of $d_{l m \pm}(\Omega) r^{l}$ functions are simple and well behaved at any $r$. For example, the second partial derivative of $d_{41-}(\Omega) r^{4}$ w.r.t. $x y$ is $L_{41-}(-6 x z)$, where $L_{41-}=0.474$ is the density function normalization factor (Paturle \& Coppens, 1988). No second derivative is more complicated than that of $d_{40}(\Omega) r^{4}$ w.r.t. $x x$, which is simply $L_{40}\left(36 x^{2}+12 y^{2}-48 z^{2}\right)$. Note that first derivatives of $d_{l m \pm}(\Omega) r^{l}$ vanish at $r=0$ for all functions except for

$$
\frac{\partial\left[d_{11+} r\right]}{\partial x}=\frac{\partial\left[d_{11-} r\right]}{\partial y}=\frac{\partial\left[d_{10} r\right]}{\partial z}=L_{1 m}=0.31831 .
$$

The second derivatives of $d_{l m \pm}(\Omega) r^{l}$ vanish at $r=0$ for all functions except for

$$
\begin{aligned}
& \frac{\partial^{2}\left[d_{21} r^{2}\right]}{\partial x \partial z}=\frac{\partial^{2}\left[d_{21-} r^{2}\right]}{\partial y \partial z}=\frac{\partial^{2}\left[d_{22} r^{2}\right]}{\partial x \partial x}=\frac{\partial^{2}\left[d_{22} r^{2}\right]}{\partial x \partial y}=L_{2 m}, \\
& \frac{\partial^{2}\left[d_{20} r^{2}\right]}{\partial x^{2}}=\frac{\partial^{2}\left[d_{20} r^{2}\right]}{\partial y^{2}}=-2 L_{20}, \\
& \frac{\partial^{2}\left[d_{20} r^{2}\right]}{\partial z^{2}}=4 L_{20}, \quad \frac{\partial^{2}\left[d_{22} r^{2}\right]}{\partial y^{2}}=-L_{22} .
\end{aligned}
$$

We define function $G(r)$ as follows:

$$
G(r)=r^{-l} I(n, l, \alpha r) .
$$

To simplify the notation of $G$, the dependence on the $n$ and $l$ indices is implicitly understood. Let

$$
j=n-l \geq 0 \text {. }
$$

The $G(r)$ function is finite and smooth at the origin and decays to zero as $r^{-2 l-1}$ for large $r$. For small $r$, the Taylor expansion 
can be used. Table 2 is equally applicable to the Taylor-series expansion of $G(r)$ and its derivatives:

$G(r)=\frac{1}{\alpha^{j+2}}\left[(j+1) !+(-1)^{j+1} \sum_{k=j+2}^{\infty} \frac{(-\alpha r)^{k}(2 l+1)}{k(k-j-2) !(2 l+1+k)}\right]$.

Alternatively,

$$
G(r)=\frac{(j+1) !}{\alpha^{j+2}}-r^{j+2} \sum_{k=0}^{\infty} \frac{(-\alpha r)^{k}(2 l+1)}{k !(k+j+2)(2 l+3+j+k)} .
$$

For example, when $n=l$ then

$$
G(r)=\frac{1}{\alpha^{2}}-r^{2}\left[\frac{(2 l+1)}{2(2 l+3)}-\frac{(\alpha r)(2 l+1)}{3(2 l+4)}+\frac{(\alpha r)^{2}(2 l+1)}{8(2 l+5)}-\ldots\right],
$$

When $n=l+1$, then

$$
G(r)=\frac{2}{\alpha^{3}}-r^{3}\left[\frac{(2 l+1)}{3(2 l+4)}-\frac{(\alpha r)(2 l+1)}{4(2 l+5)}+\frac{(\alpha r)^{2}(2 l+1)}{10(2 l+6)}-\ldots\right],
$$

Expressions (34) and (35) both have non-zero terms in $r^{3}$, which creates singularities in the second derivatives $\mathrm{d}^{2} r^{3} / \mathrm{d} q^{2}$, where $q=x, y, z$. This can be circumvented as discussed below.

Define $A(r)$ as follows:

$$
A(r)=\left(\frac{\mathrm{d} G(r)}{r \mathrm{~d} r}\right)=\frac{(-1)^{j+1}}{\alpha^{j}} \sum_{k=j}^{\infty} \frac{(-\alpha r)^{k}(2 l+1)}{(k-j) !(2 l+3+k)} .
$$

Alternatively,

$$
A(r)=-r^{j} \sum_{k=0}^{\infty} \frac{(-\alpha r)^{k}(2 l+1)}{k !(2 l+3+j+k)}
$$

Note that $A(r)$ is well behaved for small $r$ for all $l$ and $n$. Note also that (37) has a non-zero term linear in $r$ when $j=0$ or $j=1$. These linear terms have implications for $B(r)$.

Define $B(r)$ as follows:

$$
\begin{aligned}
B(r) & =\left[\frac{\mathrm{d}}{r \mathrm{~d} r}\left(\frac{\mathrm{d} G(r)}{r \mathrm{~d} r}\right)\right]=\left(\frac{\mathrm{d} A(r)}{r \mathrm{~d} r}\right) \\
& =\frac{(-1)^{j+1}}{\alpha^{j-2}} \sum_{k=j-2}^{\infty} \frac{(-\alpha r)^{k}(k+2)(2 l+1)}{(k+2-j) !(2 l+5+k)} .
\end{aligned}
$$

Alternatively,

$$
B(r)=-r^{j-2} \sum_{k=0}^{\infty} \frac{(-\alpha r)^{k}(k+j)(2 l+1)}{k !(2 l+3+j+k)} .
$$

Note that the first term vanishes in the sums (38) and (39) when $j=0$, giving

$$
B(r)=\frac{\alpha}{r} \sum_{k=0}^{\infty} \frac{(-\alpha r)^{k}(2 l+1)}{k !(2 l+4+k)}, \quad j=0 .
$$

Alternatively,

$$
B(r)=\frac{\alpha(2 l+1)}{r(2 l+4)}-\alpha^{2} \sum_{k=0}^{\infty} \frac{(-\alpha r)^{k}(2 l+1)}{(k+1) !(2 l+5+k)}, \quad j=0 .
$$

If $n=l+1$ then equation (39) can be expressed as follows:

$$
B(r)=\frac{-(2 l+1)}{r(2 l+4)}+\alpha \sum_{k=0}^{\infty} \frac{(-\alpha r)^{k}(k+2)(2 l+1)}{(k+1) !(2 l+5+k)}, \quad j=1 .
$$

The terms $r^{-1}$ in (41) and (42) require special care when $r=0$. They become infinite at the origin but are multiplied by angularly dependent factors which are zero at the origin. It turns out in these cases that the zeros are of higher order than infinity so their product is zero.

The first and partial second derivatives are then straightforward:

$$
\frac{\partial G(r)}{\partial x}=\left(\frac{\mathrm{d} G(r)}{\mathrm{d} r}\right)\left(\frac{\partial r}{\mathrm{~d} x}\right)=x A(r)
$$

Similarly,

$$
\frac{\partial G(r)}{\partial y}=y A(r)
$$

and

$$
\frac{\partial^{2} G(r)}{\partial x^{2}}=\left(\frac{\partial[x A(r)]}{\partial x}\right)=A(r)+x^{2} B(r) .
$$

Similarly,

$$
\frac{\partial^{2} G(r)}{\partial x \partial y}=\left(\frac{\partial[x A(r)]}{\partial y}\right)=x y B(r) .
$$

These formulae should be used for small $\alpha r$.

At $r=0$, derivatives are much simplified:

$$
\begin{aligned}
G(0) & =\frac{(j+1) !}{\alpha^{j+2}} \\
\left(\frac{\partial G(r)}{\partial x}\right)_{0} & =\left(\frac{\partial G(r)}{\partial y}\right)_{0}=\left(\frac{\partial G(r)}{\partial z}\right)_{0}=0 \\
\left(\frac{\partial^{2} G(r)}{\partial x^{2}}\right)_{0} & =\left(\frac{\partial^{2} G(r)}{\partial y^{2}}\right)_{0}=\left(\frac{\partial^{2} G(r)}{\partial z^{2}}\right)_{0} \\
& =A(0)= \begin{cases}\frac{-(2 l+1)}{(2 l+3)} & \text { when } j=0 \\
0 & \text { when } j>0\end{cases} \\
\left(\frac{\partial G(r)}{\partial x \partial y}\right)_{0} & =\left(\frac{\partial G(r)}{\partial x \partial z}\right)_{0}=\left(\frac{\partial G(r)}{\partial y \partial z}\right)_{0}=0 .
\end{aligned}
$$

\section{Applications of the method}

Calculations of electrostatic properties from the HansenCoppens pseudoatom formalism were performed using the newly derived formulae encoded in the new version of $X D P R O P$, part of the $X D$ package. To test the new formulae both theoretical and experimental data were used.

\subsection{ESP, EF and EFG at nuclear positions in the formamide molecule from theoretical data}

In a first example, a formamide molecule with a geometry extracted from the crystal of formamide (Stevens, 1978) was 
Table 3

ESP and vector components of the EF at the nuclear positions in formamide (atomic units) from different methods.

All components in this and the following tables refer to a global Cartesian coordinate system (atomic coordinates listed in Table 6, Appendix $A$ )

\begin{tabular}{|c|c|c|c|c|}
\hline & & Electric & & \\
\hline & Potential & $X$ & $Y$ & $Z$ \\
\hline $\mathrm{O}(1)$ & & & & \\
\hline $\mathrm{PBE} / 6-31 \mathrm{G}^{* *}$ & -22.34 & -0.096 & -0.051 & 0.001 \\
\hline PBE/aug-cc-pVDZ & -22.35 & -0.065 & -0.033 & 0.001 \\
\hline PBE/aug-cc-pVTZ & -22.36 & -0.031 & -0.016 & 0.000 \\
\hline $\mathrm{XD} / \mathrm{PBE} / 6-31 \mathrm{G}^{* *}$ & -22.42 & -0.274 & -0.174 & 0.004 \\
\hline $\mathrm{N}(2)$ & & & & \\
\hline $\mathrm{PBE} / 6-31 \mathrm{G}^{* *}$ & -18.30 & -0.002 & -0.001 & -0.001 \\
\hline PBE/aug-cc-pVDZ & -18.32 & -0.004 & 0.001 & -0.001 \\
\hline PBE/aug-cc-pVTZ & -18.32 & -0.005 & 0.002 & -0.001 \\
\hline $\mathrm{XD} / \mathrm{PBE} / 6-31 \mathrm{G}^{* *}$ & -18.44 & -0.019 & -0.009 & 0.000 \\
\hline $\mathrm{C}(3)$ & & & & \\
\hline $\mathrm{PBE} / 6-31 \mathrm{G}^{* *}$ & -14.65 & -0.019 & 0.006 & 0.001 \\
\hline PBE/aug-cc-pVDZ & -14.66 & -0.015 & 0.010 & 0.002 \\
\hline PBE/aug-cc-pVTZ & -14.67 & -0.013 & -0.001 & 0.001 \\
\hline $\mathrm{XD} / \mathrm{PBE} / 6-31 \mathrm{G} * *$ & -14.76 & -0.026 & 0.001 & 0.000 \\
\hline $\mathrm{H}(4)$ & & & & \\
\hline $\mathrm{PBE} / 6-31 \mathrm{G}^{* *}$ & -1.00 & 0.021 & 0.006 & 0.000 \\
\hline PBE/aug-cc-pVDZ & -0.98 & 0.027 & 0.011 & 0.000 \\
\hline PBE/aug-cc-pVTZ & -0.99 & 0.018 & 0.004 & 0.000 \\
\hline $\mathrm{XD} / \mathrm{PBE} / 6-31 \mathrm{G}^{* *}$ & -1.00 & 0.013 & -0.010 & 0.000 \\
\hline $\mathrm{H}(5)$ & & & & \\
\hline $\mathrm{PBE} / 6-31 \mathrm{G}^{* *}$ & -1.00 & 0.005 & -0.017 & 0.000 \\
\hline PBE/aug-cc-pVDZ & -0.98 & 0.006 & -0.024 & 0.000 \\
\hline PBE/aug-cc-pVTZ & -0.99 & 0.005 & -0.013 & 0.000 \\
\hline $\mathrm{XD} / \mathrm{PBE} / 6-31 \mathrm{G}^{* *}$ & -1.02 & 0.001 & 0.010 & 0.000 \\
\hline $\mathrm{H}(6)$ & & & & \\
\hline PBE/6-31G** & -1.10 & 0.005 & 0.026 & -0.001 \\
\hline PBE/aug-cc-pVDZ & -1.07 & 0.005 & 0.031 & -0.001 \\
\hline PBE/aug-cc-pVTZ & -1.09 & 0.006 & 0.021 & -0.001 \\
\hline $\mathrm{XD} / \mathrm{PBE} / 6-31 \mathrm{G}^{* *}$ & -1.09 & 0.003 & 0.022 & -0.007 \\
\hline
\end{tabular}

chosen. Theoretical calculations were performed with the Gaussian03 (2004) suite of programs at the density-functional (Hohenberg \& Kohn, 1964) level of theory using the 1996 exchange and correlation functionals of Perdew, Burke \& Ernzerhof $(1996,1997)$ (PBE) and 6-31G** (Hariharan \& Pople, 1973), aug-cc-pVDZ (Dunning, 1989; Kendall et al., 1992) and aug-cc-pVTZ (Dunning, 1989; Kendall et al., 1992) series of basis sets (labeled as PBE/6-31G**, PBE/aug-ccpVDZ and PBE/aug-cc-pVTZ, respectively).

For the $\mathrm{PBE} / 6-31 \mathrm{G}^{* *}$ calculation, complex static valenceonly structure factors in the range of $0<\sin \theta / \lambda<1.1 \AA^{-1}$ were obtained by analytic Fourier transform of the molecular charge densities for reciprocal-lattice points corresponding to a pseudocubic cell with $30 \AA$ edges. These data were fitted in terms of pseudoatom parameters as given in the HansenCoppens pseudoatom model [equation (8)] using the $X D$ program suite (Koritsanszky et al., 2003). Phases of all reflections were reset to the theoretical values after each refinement cycle. Both radial screening factors $\left(\kappa, \kappa^{\prime}\right)$ were refined independently for each atom, with the exception of the chemically equivalent $\mathrm{H}$ atoms which shared the same $\kappa$ and $\kappa^{\prime}$ parameters. The multipolar expansion was truncated at the hexadecapolar level $\left(l_{\max }=4\right)$ for the non- $\mathrm{H}$ atoms and at the
Table 4

Components of the EFG tensor at the nuclear positions in formamide (atomic units) from different methods.

\begin{tabular}{lcccccc}
\hline & $X X$ & $X Y$ & $X Z$ & $Y Y$ & $Y Z$ & $Z Z$ \\
\hline O(1) & & & & & & \\
PBE/6-31G** & -1216 & -1.02 & 0.02 & -1215 & 0.015 & -1216 \\
PBE/aug-cc-pVDZ & -1246 & -1.00 & 0.02 & -1245 & 0.014 & -1246 \\
PBE/aug-cc-pVTZ & -1248 & -1.00 & 0.02 & -1247 & 0.014 & -1249 \\
XD/PBE/6-31G*** & -1306 & -0.80 & -0.03 & -1305 & 0.047 & -1306 \\
N(2) & & & & & & \\
PBE/6-31G** & -798 & -0.023 & -0.032 & -798 & -0.004 & -797 \\
PBE/aug-cc-pVDZ & -819 & -0.024 & -0.031 & -819 & -0.004 & -818 \\
PBE/aug-cc-pVTZ & -821 & -0.018 & -0.031 & -821 & -0.003 & -819 \\
XD/PBE/6-31G*** & -863 & -0.015 & -0.012 & -864 & -0.013 & -863 \\
C(3) & & & & & & \\
PBE/6-31G** & -492 & 0.050 & 0.000 & -492 & 0.001 & -493 \\
PBE/aug-cc-pVDZ & -505 & 0.053 & 0.000 & -505 & 0.000 & -506 \\
PBE/aug-cc-pVTZ & -507 & 0.055 & 0.000 & -507 & 0.001 & -507 \\
XD/PBE/6-31G*** & -534 & 0.048 & -0.002 & -534 & -0.012 & -535 \\
H(4) & & & & & & \\
PBE/6-31G** & -1.95 & -0.28 & -0.012 & -1.78 & -0.008 & -1.49 \\
PBE/aug-cc-pVDZ & -1.83 & -0.28 & -0.012 & -1.66 & -0.008 & -1.38 \\
PBE/aug-cc-pVTZ & -2.00 & -0.28 & -0.012 & -1.83 & -0.008 & -1.55 \\
XD/PBE/6-31G*** & -2.29 & -0.30 & -0.012 & -2.09 & -0.009 & -1.82 \\
H(5) & & & & & & \\
PBE/6-31G** & -1.54 & 0.048 & -0.002 & -2.11 & 0.002 & -1.47 \\
PBE/aug-cc-pVDZ & -1.43 & 0.048 & -0.001 & -2.00 & 0.002 & -1.37 \\
PBE/aug-cc-pVTZ & -1.59 & 0.048 & -0.001 & -2.16 & 0.002 & -1.53 \\
XD/PBE/6-31G*** & -1.87 & 0.049 & -0.001 & -2.46 & 0.002 & -1.82 \\
H(6) & & & & & & \\
PBE/6-31G** & -1.68 & 0.009 & 0.000 & -2.11 & -0.008 & -1.66 \\
PBE/aug-cc-pVDZ & -1.50 & 0.011 & 0.000 & -1.96 & -0.008 & -1.48 \\
PBE/aug-cc-pVTZ & -1.72 & 0.010 & 0.000 & -2.15 & -0.007 & -1.71 \\
XD/PBE/6-31G*** & -2.00 & 0.007 & -0.001 & -2.45 & -0.003 & -1.99 \\
\hline & & & & & &
\end{tabular}

quadrupolar level $\left(l_{\max }=2\right)$ for $\mathrm{H}$ atoms, for which only bonddirected functions of $l, m=1,0$ and 2, 0 were refined. In order to reduce the number of least-squares variables, the following local-symmetry constraints were imposed: $m m 2$ symmetry for $\mathrm{N}$ and $m$ symmetry for $\mathrm{O}$ and $\mathrm{C}$ atoms. A molecular electroneutrality constraint was also applied. Refinement of valenceonly structure factors yield an $R$ factor of $3 \%$, with a ratio of the number of calculated structure factors to the number of refined variables of 10502 .

Tables 3-5 list the ESP, EF and EFG values at the nuclear positions as obtained directly from theoretical calculations and from the pseudoatom model, the latter labeled XD/PBE/ $6-31 \mathrm{G}^{* *}$. Electrostatic properties at the nuclear positions from theoretical data were calculated with the Gaussian03 program. Agreement in all quantities is very good, taking into account the differences between Gaussian- and Slater-type functions and the fact that the projection of the Gaussian density onto the pseudoatom model is not perfect. Especially important is the fact that the asphericity of the EFG tensor at the nuclear positions of the $\mathrm{H}$ atoms is reproduced rather well despite small differences in each of the values. In general, the pseudoatom model gives slightly higher values of all properties at the nuclear positions compared to the Gaussian calculations. This is attributed to the different behavior of Gaussian- and Slater-type functions near $r=0$, as well as the imperfect fit of the theoretical data. 
Table 5

Eigenvalues of the EFG tensor at the nuclear positions in formamide (atomic units) from different methods.

\begin{tabular}{|c|c|c|c|}
\hline & $\lambda_{1}$ & $\lambda_{2}$ & $\lambda_{3}$ \\
\hline \multicolumn{4}{|l|}{$\mathrm{O}(1)$} \\
\hline $\mathrm{PBE} / 6-31 \mathrm{G}^{* *}$ & -1217 & -1216 & -1214 \\
\hline PBE/aug-cc-pVDZ & -1247 & -1246 & -1244 \\
\hline PBE/aug-cc-pVTZ & -1249 & -1249 & -1246 \\
\hline $\mathrm{XD} / \mathrm{PBE} / 6-31 \mathrm{G}^{* *}$ & -1306 & -1306 & -1305 \\
\hline \multicolumn{4}{|l|}{$\mathrm{N}(2)$} \\
\hline $\mathrm{PBE} / 6-31 \mathrm{G}^{* *}$ & -798 & -798 & -797 \\
\hline PBE/aug-cc-pVDZ & -819 & -819 & -818 \\
\hline PBE/aug-cc-pVTZ & -821 & -821 & -819 \\
\hline $\mathrm{XD} / \mathrm{PBE} / 6-31 \mathrm{G}^{* *}$ & -864 & -863 & -863 \\
\hline \multicolumn{4}{|l|}{ C(3) } \\
\hline PBE/6-31G** & -493 & -492 & -492 \\
\hline PBE/aug-cc-pVDZ & -506 & -505 & -505 \\
\hline PBE/aug-cc-pVTZ & -507 & -507 & -507 \\
\hline $\mathrm{XD} / \mathrm{PBE} / 6-31 \mathrm{G} * *$ & -535 & -534 & -534 \\
\hline \multicolumn{4}{|l|}{$\mathrm{H}(4)$} \\
\hline $\mathrm{PBE} / 6-31 \mathrm{G}^{* *}$ & -2.16 & -1.57 & -1.49 \\
\hline PBE/aug-cc-pVDZ & -2.04 & -1.45 & -1.38 \\
\hline PBE/aug-cc-pVTZ & -2.21 & -1.62 & -1.55 \\
\hline $\mathrm{XD} / \mathrm{PBE} / 6-31 \mathrm{G}^{* *}$ & -2.50 & -1.88 & -1.82 \\
\hline \multicolumn{4}{|l|}{$\mathrm{H}(5)$} \\
\hline $\mathrm{PBE} / 6-31 \mathrm{G}^{* *}$ & -2.11 & -1.54 & -1.47 \\
\hline PBE/aug-cc-pVDZ & -2.00 & -1.42 & -1.37 \\
\hline PBE/aug-cc-pVTZ & -2.17 & -1.59 & -1.53 \\
\hline $\mathrm{XD} / \mathrm{PBE} / 6-31 \mathrm{G} * *$ & -2.46 & -1.87 & -1.82 \\
\hline \multicolumn{4}{|l|}{$\mathrm{H}(6)$} \\
\hline PBE/6-31G** & -2.11 & -1.68 & -1.66 \\
\hline PBE/aug-cc-pVDZ & -1.96 & -1.50 & -1.48 \\
\hline PBE/aug-cc-pVTZ & -2.15 & -1.72 & -1.71 \\
\hline $\mathrm{XD} / \mathrm{PBE} / 6-31 \mathrm{G}^{* *}$ & -2.45 & -2.00 & -1.99 \\
\hline
\end{tabular}

\subsection{EF in the crystal structure of formamide from theoretical data}

Note that, for an isolated molecule, the electric field at each nuclear position in the solid state must be zero once equilibrium is established. However, the molecules 'extracted' from the crystal are not at equilibrium, therefore any calculation of the isolated molecule with the crystal geometry results in significant electric forces acting on the nuclei. These forces should be 'compensated' in the crystal by those of the environment generated by the packing (of course after a final relaxation of the molecular electron density).

Fig. 1 shows the projection of the electric field in the $\mathrm{O}-$ $\mathrm{C}-\mathrm{N}$ plane of the 'central' formamide molecule due to the eight nearest-neighbor molecules as found in the crystal structure of formamide. The electric field was plotted with the program PlotMTV (Toh, 1995a) using the MTV plot data format (Toh, 1995b). The contribution of the 'central' molecule to the electric field is omitted. In general, the external electric field is directed from the 'negative' part of the central molecule (i.e. the $\mathrm{O}$ atom) towards the 'positive' end (the amino group), and has nearly the same direction as the dipole moment of formamide. Indeed, molecules in crystals tend to orient themselves so as to achieve electrostatic stabilization. By the same token, the external electric field tends to polarize the central molecule so as to increase its dipole moment and enhance this electrostatic stabilization. Our own fully periodic
(Saunders et al., 1998; Gatti, 1999) calculations at the B3LYP/ 6-31G** level of theory (Becke, 1988; Lee et al., 1988; Miehlich et al., 1989; Becke, 1993) show that the molecular dipole moment of formamide is increased to approximately 5.5 Debye in the solid state from about 4 Debye for the free molecule in the crystal geometry.

\subsection{Determination of the ${ }^{57} \mathrm{Fe}^{m}$ nuclear quadrupole moment from experimental $\mathrm{X}$-ray data}

The nuclear quadrupole moment $Q\left({ }^{57} \mathrm{Fe}^{m}\right)$ cannot be directly measured because of the short lifetime of the excited nuclear state. However, $Q\left({ }^{57} \mathrm{Fe}^{m}\right)$ is directly related to the Mössbauer quadrupole splitting $\Delta E_{Q}$ :

$$
\Delta E_{Q}=\frac{1}{2} e Q\left({ }^{57} \mathrm{Fe}^{m}\right) V_{z z}\left(1+\frac{\eta^{2}}{3}\right)^{1 / 2},
$$

where $e$ is the elementary charge, $V_{z z}$ is the largest eigenvalue of the traceless EFG tensor

$$
\left|V_{z z}\right|>\left|V_{y y}\right|>\left|V_{x x}\right|
$$

and $\eta$ is the asymmetry parameter defined as

$$
\eta=\frac{V_{x x}-V_{y y}}{V_{z z}}
$$

In order to obtain the three principal components of the EFG tensor defined by expression (5), the tensor must first be converted to its traceless form and then diagonalized. Note that $\Delta E_{Q}$ and $Q\left({ }^{57} \mathrm{Fe}^{m}\right)$ are usually given in Doppler-speed units of $\mathrm{mm} \mathrm{s}^{-1}$ and $\mathrm{m}^{2}$, respectively, while the EFG tensor components are usually reported in e $\AA^{-3}$ or atomic units. The conversion between diffraction and spectroscopic units is discussed in detail by Coppens (1997, pp. 223-224).

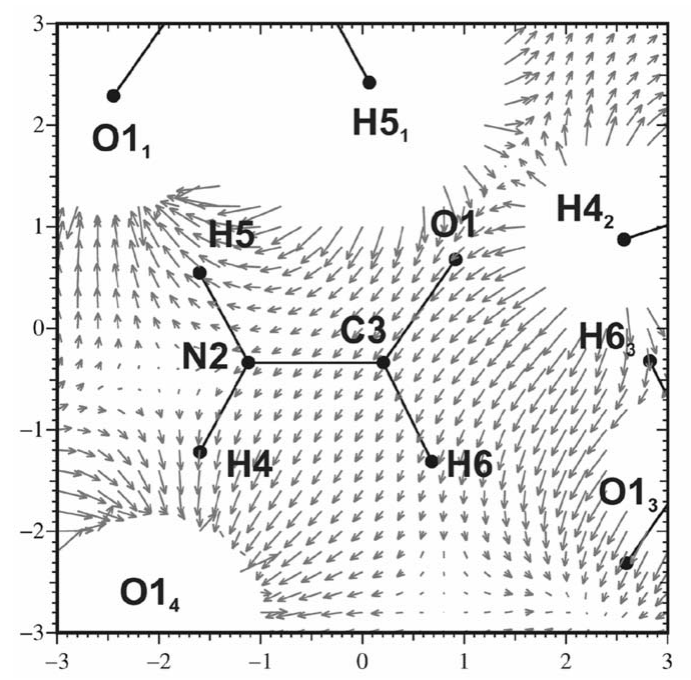

Figure 1

EF vectors in the $\mathrm{N} 2-\mathrm{C} 3-\mathrm{O} 1$ plane of the 'central' formamide molecule due to the eight nearest neighbors in the crystal (the contribution of the 'central' molecule to EF is not included). Subscripts of atom names identify the neighboring molecules. Vectors with magnitudes larger than $0.15 \mathrm{e}^{-2}$ are omitted for clarity. The size of the map is $6 \times 6 \AA$ with a grid spacing of $0.2 \AA$. 
Experimental X-ray data for iron pentacarbonyl $\mathrm{Fe}(\mathrm{CO})_{5}$ were taken from the recent low-temperature (100 K) study of Farrugia \& Evans (2005). Following the procedure of Su \& Coppens (1996), the EFG tensor at the Fe nucleus is partitioned into its peripheral $E_{\alpha \beta}^{\text {peripheral }}$ and central $E_{\alpha \beta}^{\text {central }}$ contributions:

$$
E_{\alpha \beta}(\mathbf{r})=E_{\alpha \beta}^{\text {central }}(\mathbf{r})+E_{\alpha \beta}^{\text {peripheral }}(\mathbf{r})
$$

The central component $E_{\alpha \beta}^{\text {central }}$ includes only the contribution of the Fe pseudoatom, while the peripheral component $E_{\alpha \beta}^{\text {peripheral }}$ includes both electronic and nuclear contributions of all other atoms. Because the Hansen-Coppens pseudoatom formalism uses a flexible valence shell but assumes a frozen core configuration, it is important to include the Sternheimer functions (Sternheimer, 1986) $\gamma_{\infty}^{\text {core }}$ and $R^{\text {core }}$ in order to properly describe the shielding/antishielding of the EFG at the nuclear position due to the polarization induced in the atomic density by the quadrupolar components of the density distribution (Su \& Coppens, 1996). Sternheimer functions are therefore included in expression (54) as

$$
E_{\alpha \beta}(\mathbf{r})=E_{\alpha \beta}^{\text {central }}(\mathbf{r})\left(1-R^{\text {core }}\right)+E_{\alpha \beta}^{\text {peripheral }}(\mathbf{r})\left(1-\gamma_{\infty}^{\text {core }}\right) .
$$

Values of $R^{\text {core }}=0.0730$ and $\gamma_{\infty}^{\text {core }}=-8.933$ as derived by Su \& Coppens (1996) for the neutral $\mathrm{Fe}$ atom are used in the present study.

The recent experimental value of $\Delta E_{Q}=+2.51 \mathrm{~mm} \mathrm{~s}^{-1}$ in $\mathrm{Fe}(\mathrm{CO})_{5}$ was taken as the reference. This value agrees very well with the theoretical value of $2.54 \mathrm{~mm} \mathrm{~s}^{-1}$ obtained from density-functional (B3LYP) calculations performed on the gas-phase optimized geometry of $\mathrm{Fe}(\mathrm{CO})_{5}$ (Halvin et al., 1998; Zhang et al., 2002). The reference value gives $Q\left({ }^{57} \mathrm{Fe}^{m}\right)=$ $0.11 \times 10^{-28} \mathrm{~m}^{2}$ without Sternheimer correction and $0.12 \times 10^{-28} \mathrm{~m}^{2}$ after taking into account shielding/antishielding effects of the core. These values agree very well with those previously reported by $\mathrm{Su} \&$ Coppens for iron pyrite $\mathrm{FeS}_{2}$, sodium nitroprusside $\mathrm{Na}_{2}\left[\mathrm{Fe}(\mathrm{NO})(\mathrm{CN})_{5}\right] \cdot 2 \mathrm{H}_{2} \mathrm{O}$ and $\mathrm{Fe}(\mathrm{TPP})(\text { pyridyl })_{2}$, but slightly smaller than the earlier value of $0.14(2) \times 10^{-28} \mathrm{~m}^{2}$ determined (omitting shielding/antishielding effects of the core) by Tsirel'son et al. (1987), based on studies of sodium nitroprusside and $\mathrm{Fe}_{2} \mathrm{O}_{3}$. By averaging over three compounds, Su \& Coppens obtained values of $Q\left({ }^{57} \mathrm{Fe}^{m}\right)=0.09-0.10 \times 10^{-28} \mathrm{~m}^{2}$ and $0.11-0.12 \times 10^{-28} \mathrm{~m}^{2}$ from uncorrected and corrected calculations, respectively. When our value is included in the average, the mean corrected value of $Q\left({ }^{57} \mathrm{Fe}^{m}\right)$ becomes $0.12 \times 10^{-28} \mathrm{~m}^{2}$ with a standard uncertainty of 0.02 . It is within three standard uncertainties of the most precise up-to-date determination of $Q\left({ }^{57} \mathrm{Fe}^{m}\right)=$ $0.16 \pm 5 \% \times 10^{-28} \mathrm{~m}^{2}$ reported by Dufek et al. (1995) based on the comparison of spectroscopic values with EFG's from linearized augmented-plane-wave (LAPW) theoretical densities on a series of solids. It is interesting to note that all four recent experimental X-ray charge-density studies consistently show lower values of $Q\left({ }^{57} \mathrm{Fe}^{m}\right)$ than combined theoretical/ spectroscopic studies. This discrepancy merits further study.

\section{Concluding remarks}

New practical formulae for calculation of the electrostatic potential (ESP), electric field (EF) and the electric field gradient (EFG) from the aspherical pseudoatom model are presented, which allow computation in regions near the nuclear centers. As real spherical harmonic density functions $d_{l m \pm}(\Omega)$ are discontinuous at the origin and thus non-differentiable, direct implementation of expressions containing $d_{l m \pm}(\Omega) I(n, l, \alpha r)$ in the evaluation of the electrostatic potential and its derivatives in this region is not possible. Instead the expressions have been reformulated in the form $\left[d_{l m \pm}(\Omega) r^{l}\right]\left[r^{-l} I(n, l, \alpha r)\right]$, which eliminates this problem. Special care is required when treating the integral $r^{-l} I(n, l, \alpha r)$ when $n=l$ or $n=l+1$.

The expressions are applied to a theoretical density of formamide and to the derivation of the ${ }^{57} \mathrm{Fe}^{m}$ nuclear quadrupolar moment from experimental X-ray diffraction data. For formamide, the ESP, EF and EFG at the nuclear positions, calculated with the new expressions and a projection of PBE/ 6-31G** densities onto the Hansen-Coppens pseudoatom model, agree very well with theoretical values calculated directly from the wavefunction. Small differences observed are attributed to the different behavior of Slater- and Gaussian-type functions as $r \rightarrow 0$ and to imperfections in the fitting procedure.

The new expressions have further been applied in the detailed visualization of the electric field exerted on the 'central' formamide molecule by the crystal environment. This was simulated by the electric fields of the eight closest neighboring molecules and omitting the contribution of the 'central' molecule. The direction of the EF in the central molecule almost exactly coincides with the direction of the molecular dipole moment of the formamide molecule before its incorporation into the crystal, demonstrating the importance of the electrostatic forces in determining the crystal packing. The coincidence of the dipole moment and electric field directions provides a direct explanation for the enhancement of molecular dipole moments in crystals in accord with results of numerous experimental and theoretical studies.

Determination of the nuclear quadrupole moment of iron from the experimental X-ray diffraction data of $\mathrm{Fe}(\mathrm{CO})_{5}$ yields a value of $Q\left({ }^{57} \mathrm{Fe}^{m}\right)=0.12 \times 10^{-28} \mathrm{~m}^{2}$, after taking into account shielding/antishielding effects of the core, which is in excellent agreement with previous X-ray studies by $\mathrm{Su} \&$ Coppens (1996). However, this value is slightly smaller than the generally accepted value of $0.16 \pm 5 \% \times 10^{-28} \mathrm{~m}^{2}$ obtained from combined theoretical/spectroscopic studies (Dufek et al., 1995). The fact that X-ray determinations of $Q\left({ }^{57} \mathrm{Fe}^{m}\right)$ using different crystals and data sets consistently yield slightly lower values than those obtained from theoretical and spectroscopic studies requires further examination.

Note that the formulae presented for calculation of the ESP have already been used in our previous studies on the calculation of the electrostatic interaction energies in molecular crystals (Volkov et al., 2004, 2006). Numerical quadrature 
evaluation of the two-centered Coulomb integral in the exact potential and multipole moment (EPMM) method requires an accurate yet efficient evaluation of the ESP at any $r$.

Application of the new method to topological analysis of the ESP, as recently performed by Bouhmaida et al. (2002), is currently being pursued.

\section{APPENDIX $A$}

Table 6

Cartesian coordinates $(\AA)$ of the atoms in formamide used in all calculations.

\begin{tabular}{lrrr}
\hline Atom & \multicolumn{1}{c}{$y$} & \multicolumn{1}{l}{$z$} \\
\hline $\mathrm{O}(1)$ & -1.1985900 & -0.2390700 & 0.0036000 \\
$\mathrm{~N}(2)$ & 1.0736400 & -0.1651500 & -0.0015000 \\
$\mathrm{C}(3)$ & -0.1305900 & 0.3877800 & -0.0088500 \\
$\mathrm{H}(4)$ & 1.8732000 & 0.4369500 & 0.0209100 \\
$\mathrm{H}(5)$ & 1.1409300 & -1.1690400 & 0.0014700 \\
$\mathrm{H}(6)$ & -0.1574100 & 1.4741700 & 0.0124500 \\
\hline
\end{tabular}

Financial support of this work by the National Science Foundation (CHE0236317) is gratefully acknowledged.

\section{References}

Becke, A. D. (1988). Phys. Rev. A, 38, 3098-3100.

Becke, A. D. (1993). J. Chem. Phys. 98, 5648-5652.

Bertaut, E. F. (1978). J. Phys. Chem. Solids, 39, 97-102.

Bouhmaida, N., Dutheil, M., Ghermani, N. E. \& Becker, P. (2002) J. Chem. Phys. 116, 6196-6204.

Bouhmaida, N., Ghermani, N. E., Lecomte, C. \& Thalal A. (1997). Acta Cryst. A53, 556-563.

Brown, A. S. \& Spackman, M. A. (1994). Mol. Phys. 83, 551-566.

Clementi, E. \& Roetti, C. (1974). At. Data Nucl. Data Tables, 14, 177-478.

Coppens, P. (1997). X-ray Charge Densities and Chemical Bonding. New York: Oxford University Press.

Dufek, P., Blaha, P. \& Schwarz, K. (1995). Phys. Rev. Lett. 25, 3545-3548.

Dunning, T. H. Jr (1989). J. Chem. Phys. 90, 1007-1023.

Edmonds, A. R. (1974). Angular Momentum in Quantum Mechanics. New Jersey: Princeton University Press.

Epstein, J. \& Swanton, D. J. (1982). J. Chem. Phys. 77, 1048-1060.

Farrugia, L. J. \& Evans, C. (2005). J. Phys. Chem. A109, 8834-8848.

Gatti, C. (1999). TOPOND98 Users' Manual, CNR-CSRSRC, Milano, Italy.

Gaussian 03 (2004). Gaussian 03, Revision C.02, by M. J. Frisch, G. W. Trucks, H. B. Schlegel, G. E. Scuseria, M. A. Robb, J. R. Cheeseman, J. A. Montgomery Jr, T. Vreven, K. N. Kudin, J. C. Burant, J. M. Millam, S. S. Iyengar, J. Tomasi, V. Barone, B. Mennucci et al. Gaussian, Inc., Wallingford, CT, USA.

Ghermani, N. E., Bouhmaida, N. \& Lecomte, C. (1992). ELECTROS: Computer Program to Calculate Electrostatic Properties from High Resolution X-ray Diffraction. Internal Report URA CNRS 809. Université de Nancy I, France.

Ghermani, N. E., Bouhmaida, N. \& Lecomte, C. (1993). Acta Cryst A49, 781-789.
Ghermani, N. E., Bouhmaida, N., Lecomte, C., Papet, A.-L. \& Marsura, A. (1994). J. Phys. Chem. 98, 6287-6292.

Ghermani, N. E., Lecomte, C. \& Bouhmaida, N. (1993). Z. Naturforsch. 48, 91-98.

Halvin, R. H., Godbout, N., Salzman, R., Wojdelski, M., Arnold, W., Schulz, C. E. \& Oldfield, E. (1998). J. Am. Chem. Soc. 120, 3144-3151.

Hansen, N. K. \& Coppens, P. (1978). Acta Cryst. A34, 909-921.

Hariharan, P. C. \& Pople, J. A. (1973). Theor. Chim Acta, 28, 213-222.

He, X. M. (1983). The MOLPOT Computer Program. Tech. Rep., Department of Crystallography, University of Pittsburgh, PA, USA.

Hohenberg, P. \& Kohn, W. (1964). Phys. Rev. 136, B864.

Jackson, J. D. (1975). Classical Electrodynamics. New York: John Wiley and Sons, Inc.

Kendall, R. A., Dunning, T. H. Jr \& Harrison, R. J. (1992). J. Chem. Phys. 96, 6796-6806.

Koritsanszky, T., Howard, S. T., Richter, T., Macchi, P., Volkov, A., Gatti, C., Mallinson, P. R., Farrugia, L. J., Su, Z. \& Hansen, N. K (2003). XD - a Computer Program Package for Multipole Refinement and Topological Analysis of Charge Densities from Diffraction Data. Middle Tennessee State University, TN, USA; University of Milano, Italy; University at Buffalo, NY, USA; CNR-ISTM, Milano, Italy; University of Glasgow, UK.

Lecomte, C., Ghermani, N., Pichon-Pesme, V. \& Souhassou, M. (1992). J. Mol. Struct. (Theochem), 255, 241-260.

Lee, C., Yang, W. \& Parr, R. G. (1988). Phys. Rev. B, 37, 785-789.

Miehlich, B., Savin, A., Stoll, H. \& Preuss, H. (1989). Chem. Phys. Lett. 157, 200-206.

Paturle, A. \& Coppens, P. (1988) Acta Cryst. A44, 6-8.

Perdew, J. P., Burke, K. \& Ernzerhof, M. (1996). Phys. Rev. Lett. 77, 3865-3868.

Perdew, J. P., Burke, K. \& Ernzerhof, M. (1997). Phys. Rev. Lett. 78, 1396-1396.

Saunders, V. R., Dovesi, R., Roetti, C., Causà, M., Harrison, N. M., Orlando, R. \& Zicovich-Wilson, C. M. (1998). CRYSTAL98 User's Manual, University of Torino, Italy.

Schwarzenbach, D. \& Thong, N. (1979). Acta Cryst. A35, 652-658.

Slater, J. C. (1932). Phys. Rev. 42, 33-43.

Sternheimer, R. M. (1986). Z. Naturforsch. Teil A, 41, 24-36.

Stevens, E. D. (1978). Acta Cryst. B34, 544-551.

Stewart, R. F. (1979). Chem. Phys. Lett. 65, 335-342.

Stewart, R. F., Spackman, M. A. \& Flensburg, C. (1998). VALRAY98, Users Manual, Carnegie Mellon University, Pittsburgh, PA, USA, and University of Copenhagen, Denmark.

Su, Z. \& Coppens, P. (1992). Acta Cryst. A48, 188-197.

Su, Z. \& Coppens, P. (1996). Acta Cryst. A52, 748-756.

Toh, K. K. H. (1995a). PlotMTV - Fast Multi-Purpose Plotting Program for X11-Windows.

Toh, K. K. H. (1995b). MTV Plot Data Format, Version 1.4.1, Rev. 0.

Tsirel'son, V. G., Strel'tsov, V. A., Makarov, E. F. \& Ozerov, R. P. (1987). Sov. Phys. JETP, 65, 1065-1069.

Velde, B. te (1990). BAND - a Fortran Program for Band Structure Calculations. PhD thesis, Vrije Universiteit, Amsterdam, The Netherlands.

Velde, B. te, Bickelhaupt, F. M., van Gisbergen, S. J. A., Fonseca Guerra, C., Baerends, E. J., Snijders, J. G. \& Ziegler, T. J. (2001). J. Comput. Chem. 22, 931-967.

Volkov, A., King, H. F. \& Coppens, P. (2006). J. Chem. Theory Comput. 2, 81-89.

Volkov, A., Koritsanszky T. S. \& Coppens, P. (2004). Chem. Phys. Lett. 391, 170-175.

Zhang, Y., Mao, J., Godbout, N. \& Oldfield, E. (2002). J. Am. Chem. Soc. 124, 13921-13930. 
Acta Crystallographica Section A

Foundations of

Crystallography

ISSN 0108-7673

Received 17 October 2006

Accepted 11 January 2007

\section{Comment on On the calculation of the electrostatic potential, electric field and electric field gradient from the aspherical pseudoatom model by Volkov, King, Coppens \& Farrugia (2006)}

\author{
Mark A. Spackman \\ Chemistry - M313, School of Biomedical, Biomolecular \& Chemical Sciences, University of Western Australia, \\ Crawley, WA 6009, Australia. Correspondence e-mail: mas@cyllene.uwa.edu.au
}

Printed in Singapore - all rights reserved

incorporating vibrational (or thermal) averaging of deformation electron densities closely approximate static results in regions far from the nuclei.

A number of papers that emerged from Stewart's group in the early 1980s outlined Fourier summation, direct space and combined strategies for the computation of the ESP, EF and EFG, with examples drawn from pseudoatom multipole fits to experimental data for imidazole and 9-methyladenine measured by Craven's group at the University of Pittsburgh (Spackman \& Stewart, 1981; Stewart, 1982; Spackman \& Stewart, 1984). Later applications of this kind included a combined Fourier/direct-space approach to mapping the total ESP in sodium zeolite A (Spackman \& Weber, 1988), and a detailed presentation of ESP maps for molecules and molecular clusters of urea, imidazole, 9-methyladenine and benzene (Stewart, 1991). All of these results were based on algorithms developed by Stewart that were unfortunately not published at the time. They were incorporated into early versions of $V A L R A Y$, and the 1983 version of $V A L R A Y$ implemented the entire suite of Fourier, direct-space and combined approaches to the computation of the ESP, EF and EFG for isolated molecules, clusters or the crystal. Stewart's expressions for the direct-space computation of the ESP from the parameters of the pseudoatom model were also incorporated into MOLPOT, part of the series of programs known as the 'POP procedure' (He, 1984; Craven et al., 1987; Craven, 1988). MOLPOT in turn formed the basis for ELECTROS (Ghermani et al., 1992), and Ghermani et al. (1993) have published an independent derivation of the relevant expressions for the ESP.

Su \& Coppens (1992) published the first complete derivation and expressions for computing not only the ESP but also the EF and EFG from pseudoatom model parameters, including penetration (peripheral) contributions, based on a generalization of the Fourier convolution approach used by Epstein \& Swanton (1982). Their expressions were exceedingly complex, and according to VKCF involved 'the evaluation of fairly complicated one-electron twocentre integrals', and further comments in the recent paper suggest that their incorporation into the $X D$ suite was never fully satisfactory. The VKCF paper presents a careful review of the derivation of the expressions published by Ghermani et al. (1993) and, instead of the Green function approach used by Ghermani et al., VKCF expand $\left|\mathbf{r}-\mathbf{r}^{\prime}\right|^{-1}$ directly in real spherical harmonics, along the lines of previous work (Bentley, 1981; te Velde, 1990; De Bondt et al., 1993; te Velde et al., 2001).

Stewart's detailed derivation for the ESP was published in conjunction with Craven in a study on $\gamma$-aminobutyric acid (Stewart \& Craven, 1993). It presents Stewart's beautiful and elegant derivation of the same expressions published by others, including the closed 
forms of equations (22) and (23) in VKCF, and based on the Fourier convolution approach. The key difference between this and other derivations is that Stewart's makes use of his intimate knowledge of Bessel functions and their inter-relationships. This is more than a mathematical curiosity; it enabled relatively straightforward extension of the same approach to the derivation of expressions for the EF and EFG, yielding separate expressions for all three components of the EF, and for all five components of the traceless EFG tensor. As noted above, these latter derivations and expressions were never published in the peer-reviewed literature, but they were incorporated in Appendix C of the VALRAY manual in 2000 (Stewart et al., 2000). As that manual has not been widely circulated, in the interests of wider dissemination of those important results, that Appendix is provided in PDF format as supplementary material accompanying this communication. ${ }^{\mathbf{1}}$

This Letter concludes with some remarks pertaining to the results and discussion in $\S 4$ of the paper by VKCF. The first concerns the EF, and in particular the map of EF projected onto the plane of the formamide molecule (Fig. 1 of VKCF), based on a pseudoatom modelling of theoretical structure factors. The contribution of the 'central' molecule has been omitted from the map and, as VKCF emphasize, the resulting map very nicely demonstrates how the 'central' molecule experiences an EF due to surrounding molecules that is closely parallel to its own dipole moment vector. This is an important result, and the strategy used to obtain it, namely subtracting the contribution from a particular molecule (or atom or ion), deserves to be used more widely. In light of the comments above, it is worth noting that the map presented by VKCF is based on a direct-space summation of contributions from only the eight nearest neighbours in the crystal. The computation of the EF in the crystal due to point dipoles is well known to require lattice summation techniques to achieve convergence (Cummins et al., 1976), and that is one reason why Stewart advocated combined Fourier/direct-space approaches to computing some properties, and these combined approaches are entirely analogous to the Ewald approach used to achieve rapid convergence of lattice sums. Thus, computation of the ESP and EF in the crystal are best achieved with an approach involving both summation over appropriate Fourier coefficients (e.g. those due to the deformation density) as well as a direct-space calculation (e.g. over spherical atoms, including nuclei).

Regarding the EFG, VKCF write (p. 401): 'Several methods for the calculation of the ESP/EFG were proposed by Brown \& Spackman (1994)', and 'While giving more or less reasonable results, these methods are either too computationally demanding or have convergence problems with Fourier sums.' These comments rather miss the point of the calculations presented in that paper. We noted in that paper that, at that time, the direct-space EFG code in VALRAY had never been tested fully, and the purpose of the various computational routes to the EFG was simply to validate that code in VALRAY (not the algorithms). Thus, we compared results and convergence behaviour of the direct-space EFG with: (i) a combined Fourier/directspace approach as described above for the EF; and (ii) numerical second differentiation of both the direct-space and combined Fourier/ direct-space approaches to the ESP. It was never our intention that any approach other than the direct-space calculation be used to compute the EFG from the pseudoatom model; we did locate minor bugs and proceeded to validate the corrected code.

\footnotetext{
${ }^{1}$ Supplementary material for this paper is available from the IUCr electronic archives (Reference: SH5054). Services for accessing this material are described at the back of the journal.
}

Also on p. 401, VKCF state that 'the method of Su \& Coppens only allows calculation of the traceless EFG tensor at the nuclear positions'. Equation (1) of their paper, Poisson's equation, relates the Laplacian of the ESP to the electron density, and hence any code that computes the electron density and the traceless EFG can also provide the complete EFG tensor, although only the traceless EFG tensor is relevant in the computation of quantities such as nuclear quadrupole coupling constants measured in NMR or NQR experiments (Cohen \& Reif, 1957). In their comparison between EFG results derived from pseudoatom modelling of theoretical structure factors for formamide (labelled $\mathrm{XD} / \mathrm{PBE} / 6-31 \mathrm{G}^{* *}$ in their tables) and those directly from the $a b$ initio wavefunction (labelled PBE/6-31G**), VKCF use the whole EFG tensor (i.e. not the traceless tensor), which appears to obscure any agreement between the two. Although VKCF (p. 405) conclude that agreement between the two is 'very good, taking into account the differences between Gaussian- and Slater-type functions', this reflects the systematic differences between electron densities at the nuclei. Converting their results (reported in their Tables 4 and 5) to traceless form reveals that for $\mathrm{H}$ atoms their $\mathrm{XD} /$ $\mathrm{PBE} / 6-31 \mathrm{G}^{* *}$ results are in almost perfect agreement with those obtained directly from the wavefunction, and that (to only the single significant figure available) a similar result holds for the $\mathrm{C}$ atom, but agreement gets progressively worse for $\mathrm{N}$ and $\mathrm{O}$, atoms with more contracted electron densities. These conclusions are in excellent agreement with our own detailed model studies on a number of molecular crystals (Spackman \& Byrom, 1996; Spackman et al., 1999), with our earlier work on benzene and corundum (Brown \& Spackman, 1994), and they clearly echo conclusions by Epstein et al. (1977), based on generalized scattering factor (g.s.f.) expansions for diatomic molecules.

A final comment concerns the use of Sternheimer corrections by VKCF in their computation of the EFG tensor at the Fe nucleus based on multipole modelling of X-ray data for iron pentacarbonyl. The use of Sternheimer corrections was never discussed by Stewart, nor in our own work, yet appears in analyses by Coppens and coworkers [for a detailed discussion and summary of many results, see Section 10.3 of Coppens's monograph (Coppens, 1997), and Section VII.C of the review article by Koritsanszky \& Coppens (2001)], and has been used without question in recent analyses (Dahaoui et al., 2001). However, the use of Sternheimer corrections deserves to be questioned, as they were introduced to describe so-called 'core polarization' at a time when EFGs in solids were estimated using point charges on atomic sites. VKCF incorporate both a shielding factor, $R^{\text {core }}$, and an antishielding factor, $\gamma_{\infty}$, resulting in an expression for the EFG tensor components for the Fe nucleus, $E_{\alpha \beta}(\mathbf{r})=0.927 E_{\alpha \beta}^{\text {central }}(\mathbf{r})+9.933 E_{\alpha \beta}^{\text {peripheral }}(\mathbf{r})$. Thus, the contribution from the nucleus-centred quadrupole functions is reduced by $\sim 7 \%$, and the (generally much smaller) peripheral (penetration) contribution is enhanced by an order of magnitude by including these correction factors. Tsirelson \& Ozerov (1996) have argued that the antishielding correction should only be included when the peripheral term is approximated by a point-charge model, and this makes a great deal of sense. It is also arguable that even the shielding contribution should be included. Coppens (1997) argued that its use takes into account the fact that the pseudoatom model uses a frozen core approximation, and thus cannot describe core polarization effects. However, Schwarz, Blaha and co-workers have performed accurate all-electron theoretical calculations of EFGs in a variety of complex solids using a full-potential linearized augmented plane-wave method (FP LAPW) and make no use of any Sternheimer corrections (Blaha et al., 1985; Schwarz \& Blaha, 1992; Dufek et al., 1995; Blaha et al., 2000; Schwarz \& Blaha, 2003). Most importantly, their calculations 
provide a breakdown of the EFG tensors into contributions from semi-core (SC) and valence states. For a series of $\mathrm{Al}_{2} \mathrm{SiO}_{5}$ polymorphs (Iglesias et al., 2001), they conclude that the 'main contribution to the EFG comes from the distortion from spherical symmetry of the respective valence $p$ electrons', although for $\mathrm{Al}$ and $\mathrm{Si}$ important contributions come from the low-lying semi-core states on $\mathrm{Al}(2 p)$ and $\mathrm{O}(2 s)$. Similarly, results for a series of Fe-containing solids indicate that 'usually the Fe $3 p$ SC state contribution is less than $10 \%$ of the valence part' (Dufek et al., 1995). This is not intended to suggest that core polarization is negligible, but it seems likely that more important factors currently hamper determination of accurate EFG tensors for heavy atoms from X-ray diffraction data (e.g. a complete and accurate description of the thermal motion of the nucleus, limited flexibility of radial functions in the pseudoatom model, and inherent limits on the resolution of the X-ray diffraction data), and the use of Sternheimer shielding factors is likely to obscure any attempts at improvement in these areas.

It is worth reiterating that the new expressions and algorithms presented by VKCF and especially their incorporation into $X D$ - now used in more than three-quarters of published experimental chargedensity studies - represents an important step towards the routine determination and presentation of ESP, EF and EFG results based on modern charge-density studies. In particular, it would be a suitably fitting outcome of the incorporation of the new expressions by VKCF into $X D$ if attention could be focused on the EFG tensor once again, with the aim of seeking quantitative agreement with NQR results where possible, as advocated by Stewart (1977) nearly 30 years ago.

I am most indebted to Bryan Craven and Joseph Ho (Xiao-Min $\mathrm{He}$ ) for their comments regarding the incorporation of Stewart's algorithms into the POP series of programs at the University of Pittsburgh. Thanks are also due to Henning Sørensen, who kindly confirmed for me the contents of various versions of the VALRAY manual.

\section{References}

Bentley, J. (1981). Chemical Applications of Atomic and Molecular Electrostatic Potentials, edited by P. Politzer \& D. G. Truhlar, pp. 63-84. New York: Plenum.

Bertaut, E. F. (1978). J. Phys. Chem. Solids, 39, 97-102.

Blaha, P., Schwarz, K., Faber, W. \& Luitz, J. (2000). Hyperfine Inter. 126, 389-395.

Blaha, P., Schwarz, K. \& Herzig, P. (1985). Phys. Rev. Lett. 54, 1192-1195.

Brown, A. S. \& Spackman, M. A. (1994). Mol. Phys. 83, 551-566.

Cohen, M. H. \& Reif, F. (1957). Solid State Phys. Adv. Res. Applic. 5, 321-438.

Coppens, P. (1997). X-ray Charge Densities and Chemical Bonding. New York: Oxford University Press.

Craven, B. M. (1988). Crystallographic Computing 4. Techniques and New Technologies, edited by N. W. Isaacs \& M. R. Taylor, pp. 211-220. Oxford University Press.
Craven, B. M., Weber, H.-P. \& He, X. (1987). Report TR-87-2, University of Pittsburgh, PA, USA.

Cummins, P. G., Dunmur, D. A., Munn, R. W. \& Newham, R. J. (1976). Acta Cryst. A32, 847-853.

Dahaoui, S., Ghermani, N. E., Ghose, S. \& Howard, J. A. K. (2001). Am. Mineral. 86, 159-164.

De Bondt, H. L., Blaton, N. M., Peeters, O. M. \& De Ranter, C. J. (1993). Z. Kristallogr. 208, 167-174.

Dufek, P., Blaha, P. \& Schwarz, K. (1995). Phys. Rev. Lett. 75, 3545-3548.

Epstein, J., Bentley, J. \& Stewart, R. F. (1977). J. Chem. Phys. 66, 5564-5567.

Epstein, J. \& Swanton, D. J. (1982). J. Chem. Phys. 77, 1048-1060.

Ghermani, N., Lecomte, C. \& Bouhmaida, N. (1993). Z. Naturforsch. Teil A, 48, 91-98.

Ghermani, N. E., Bouhmaida, N. \& Lecomte, C. (1992). ELECTROS: Computer Program to Calculate Electroststic Properties from High Resolution X-ray Diffraction. Internal Report URA CNRS 809, Université de Nancy I, France.

He, X. M. (1984). PhD thesis, University of Pittsburgh, PA, USA.

Iglesias, M., Schwarz, K., Blaha, P. \& Baldomir, D. (2001). Phys. Chem. Miner. 28, 67-75.

Koritsanszky, T. \& Coppens, P. (2001). Chem. Rev. 101, 1583-1627.

Koritsanszky, T., Howard, S. T., Macchi, P., Gatti, C., Farrugia, L. J., Mallinson, P. R., Volkov, A., Su, Z., Richter, T. \& Hansen, N. K. (2003). XD - A Computer Program Package for Multipole Refinement and Analysis of Charge Densities from Diffraction Data, 4.10 ed. Middle Tennessee State University, TN, USA; University of Milano, Italy; University of Glasgow, UK.

Schwarz, K. \& Blaha, P. (1992). Z. Naturforsch. Teil A, 47, 197-202.

Schwarz, K. \& Blaha, P. (2003). Comput. Mater. Sci. 28, 259-273.

Schwarzenbach, D. \& Thong, N. (1979). Acta Cryst. A35, 652-658.

Spackman, M. A. \& Byrom, P. G. (1996). Acta Cryst. B52, 1023-1035.

Spackman, M. A., Byrom, P. G., Alfredsson, M. \& Hermansson, K. (1999). Acta Cryst. A55, 30-47.

Spackman, M. A. \& Stewart, R. F. (1981). Chemical Applications of Atomic and Molecular Electrostatic Potentials, edited by P. Politzer \& D. G. Truhlar, pp. 407-425. New York: Plenum.

Spackman, M. A. \& Stewart, R. F. (1984). Methods and Applications in Crystallographic Computing, edited by S. R. Hall \& T. Ashida, pp. 302-320. Oxford: Clarendon Press.

Spackman, M. A. \& Weber, H.-P. (1988). J. Phys. Chem. 92, 794-796.

Stewart, R. F. (1977). Chem. Phys. Lett. 49, 281-284.

Stewart, R. F. (1979). Chem. Phys. Lett. 65, 335-342.

Stewart, R. F. (1982). God. Jugosl. Cent. Kristalogr 17, 1-24.

Stewart, R. F. (1991). The Application of Charge Density Research to Chemistry and Drug Design, edited by G. A. Jeffrey \& J. F. Piniella, pp. 63-102. New York: Plenum Press.

Stewart, R. F. \& Craven, B. M. (1993). Biophys. J. 65, 998-1005.

Stewart, R. F., Spackman, M. A. \& Flensburg, C. (2000). VALRAY - User's Manual, 2.1 ed. Carnegie Mellon University, Pittsburgh, PA, USA, and University of Copenhagen, Denmark.

Su, Z. \& Coppens, P. (1992). Acta Cryst. A48, 188-197.

Tsirelson, V. \& Ozerov, R. P. (1996). Electron Density and Bonding in Crystals. London: IOP Publishing.

Velde, G. te (1990). PhD thesis, Vrije Universiteit, Amsterdam, The Netherlands.

Velde, G. te, Bickelhaupt, F. M., Baerends, E. J., Fonseca Guerra, C., van Gisbergen, S. J. A., Snijders, J. G. \& Ziegler, T. (2001). J. Comput. Chem. 22, 931-967.

Volkov, A., King, H. F., Coppens, P. \& Farrugia, L. J. (2006). Acta Cryst. A62, 400-408. 